\title{
BIODEGRADABLE NANOPARTICLES OBTAINED FROM ZEIN AS A DRUG DELIVERY SYSTEM FOR TERPINEN-4-OL
}

Vanderléia G. Marinia ${ }^{a}$, Silvia M. Martellia ${ }^{\text {, Clarice F. Zornio }}{ }^{\mathrm{a}}$, Thiago Caon ${ }^{\mathrm{b}}$, Cláudia M. O. Simões ${ }^{\mathrm{b}}$, Gustavo A. Micke ${ }^{\mathrm{a}}$, Marcone A. L. de Oliveirac ${ }^{c}$, Vanderlei G. Machado ${ }^{a}$ and Valdir Soldia,*

aDepartamento de Química, Universidade Federal de Santa Catarina, Florianópolis - SC, Brasil

bepartamento de Ciências Farmacêuticas, Universidade Federal de Santa Catarina, Florianópolis - SC, Brasil

'Departamento de Química, Universidade Federal de Juiz de Fora, Juiz de Fora - MG, Brasil

Recebido em 29/10/2013; aceito em 31/01/2014; publicado na web em 08/05/2014

\begin{abstract}
Biodegradable nanoparticles (NPs) have received considerable attention because of their possible use in the development of strategies for the topical delivery of oils and therapeutic drugs, particularly when drug penetration in dermis is desired. Zein is a prolamine and is a promising material for the design of drug delivery systems. In this study, NPs were prepared with zein and were used to encapsulate and release terpinen-4-ol, which is a therapeutic agent for the treatment of melanoma. The results show that the zein NPs are promising nanostructured systems for the prolonged delivery of T4OL with potential applications in anti-melanoma therapy.
\end{abstract}

Keywords: zein nanoparticles; terpinen-4-ol; in vitro release.

\section{INTRODUCTION}

Melanoma cancer is the most dangerous type of skin cancer because it is associated with a high mortality rate. This highly invasive and metastatic tumor is usually refractory in relation to chemotherapy because melanoma cells exhibit a high level of intrinsic resistance to various traditional cytostatic agents. ${ }^{1}$ Only $4 \%$ of skin cancer cases are diagnosed as melanoma, yet this form accounts for $79 \%$ of all deaths due to skin cancer. ${ }^{2}$ Therefore, new bioactive compounds and strategies are needed to address this issue, and the development of new delivery systems to treat melanoma is an extremely important subject. Terpinen-4-ol (T4OL), which is the main component of tea tree oil and is also present in various essential oils (e.g., juniper oil, lavender oil, rosemary oil, majoram oil, and thyme oil), ${ }^{3}$ exhibits an in vitro inhibitory effect on the growth of multidrug-resistant human melanoma cells. ${ }^{4,5}$

Polymers suitable for use in skin drug delivery systems need to be stable, nonreactive with the drug, inexpensive, and easily manufactured. ${ }^{6}$ The zein storage proteins are frequently found in maize endosperm, where they are deposited as protein bodies within the rough endoplasmic reticulum. ${ }^{7,8}$ Zeins are renewable, biodegradable, biocompatible, and nontoxic. They have more hydrophobic than hydrophilic amino acids in their structure, making them insoluble in water, which is a requirement for the preparation of water-based formulations. ${ }^{9,10}$ These proteins have been extensively investigated because of their ability to form nanoparticles (NPs) for the encapsulation of bioactive compounds, such as essential oils, ${ }^{11,12}$ and also for controlled drug delivery for biomedical applications. ${ }^{13,14,10}$ Because of their high biocompatibility and ability to form nanospheres through coacervation, zeins are natural raw materials with potential uses in tissue engineering, drug delivery systems, and biomedicine. ${ }^{1,15}$

Biodegradable NPs have received considerable attention because of their potential usefulness in the development of strategies for the topical delivery of oils and therapeutic drugs, ${ }^{16-18}$ particularly when drug penetration into the dermis is desirable. ${ }^{19}$ Most delivery systems with properties suitable for controlled release are microscale beads or gels..$^{20}$ To date, encapsulation and delivery systems for nanoscale

*e-mail: soldi.valdir@gmail.com
T4OL, with the addition of zein as a carrier, have not been developed and characterized.

In the development of drug delivery systems, the release behavior is a key factor in establishing their applicability. ${ }^{21}$ Thus, the objective of this study was to optimize a biodegradable and biocompatible nanostructured system for the local delivery of T4OL. Because the skin has natural protection against the penetration of external media, ${ }^{22}$ skin penetration enhancers (SPEs) were also added to the formulations to evaluate their effect on the physicochemical properties and in vitro release of T4OL.

\section{EXPERIMENTAL}

\section{Materials}

Corn zein, T4OL, $n$-tetradecyl trimethyl ammonium bromide (TTA), $R$-(+)-limonene, oleic acid, and propylene glycol were obtained from Sigma-Aldrich. Ethanol was acquired from LAFAN, and acetonitrile was purchased from VETEC. All reagents were used as received. Phosphate-saline buffer (PBS) at $\mathrm{pH} 7.4$ was used in the experiments.

\section{Preparation and physicochemical characterization of NPs}

Zein NPs were prepared using a modified antisolvent precipitation process (desolvation). ${ }^{23}$ In this process, the zein was initially dissolved in a binary solvent of ethanol/water (87:13) and T4OL and T4OL + SPE were subsequently added. The SPEs studied were $R$-(+)-limonene, propylene glycol, and oleic acid; these SPEs were added individually to the zein solutions, which were then mixed for $30 \mathrm{~min}$. For each sample, $3 \mathrm{~mL}$ of zein solution containing T4OL was dropped at a constant rate of $6 \mathrm{~mL} \mathrm{~h}^{-1}$ into $9 \mathrm{~mL}$ of an aqueous solution containing the selected amount of TTA under magnetic stirring at $1000 \mathrm{rpm}$. The organic solvent was removed by evaporation under reduced pressure.

The particle size and zeta potential of the freshly prepared NP dispersions were determined by photon correlation spectroscopy and laser-Doppler anemometry, respectively, using a Zetasizer Nano Series analyzer (Malvern Instruments, Worcestershire, UK). 
Morphological analysis of NPs was performed using transmission electron microscopy (TEM). To calculate the encapsulation efficiency $(E E)$, we centrifuged the resulting suspensions at $4000 \mathrm{rpm}$ for 30 min using an Amicon ultra-filter (a regenerated cellulose membrane with a molecular-weight cutoff of $100 \mathrm{kDa}$ ). The supernatants were removed, diluted in $87 \%$ ethanol, and analyzed for T4OL content by HPLC with UV/vis detection. An isocratic eluent consisting of acetonitrile:Milli-Q water (55:45) and a C18 column (XDB, $4.6 \times$ $150 \mathrm{~mm}^{2}, 5 \mu \mathrm{m}$, Agilent Zorbax Eclipse) were used; the flow rate was $1.3 \mathrm{~mL} \mathrm{~min}^{-1}$, the injection volume was $20 \mu \mathrm{L}$, and the detection wavelength was $200 \mathrm{~nm}$. The $E E$ was calculated on the basis of the difference between the amount of T4OL added to the zein solution and the amount present in the supernatant (not encapsulated).

\section{In vitro release}

In vitro release experiments were performed with samples containing $50 \mathrm{mg} \mathrm{mL}^{-1}$ of zein, $50 \mathrm{mg} \mathrm{mL}^{-1}$ of T4OL, and $0.3 \%$ of TTA. $R$-(+)-Limonene, propylene glycol, or oleic acid was added as the SPE to assess their influence on the T4OL release. In vitro release experiments $(n=3)$ with synthetic membranes (Spectra/Por ${ }^{\circledR} 3$. $3500 \mathrm{~g} \mathrm{~mol}^{-1}$ ) were performed in glass Franz-type diffusion cells with a receptor volume of $10 \mathrm{~mL}$ and a diffusional area of $1.77 \mathrm{~cm}^{2}$. An isotonic PBS containing 1\% (w/v) Tween 80 was included as the receptor medium, and the system was continuously stirred. The system was maintained at $37^{\circ} \mathrm{C}$ to mimic in vivo conditions. Predefined concentrations of each formulation were separately placed in the donor compartment (zein:T4OL; zein:T4OL:SPEs). At time intervals of $1,2,3,4,5,6$, and $7 \mathrm{~h}, 400 \mu \mathrm{L}$ of each sample was removed from the receptor and immediately replaced with fresh PBS/Tween 80 solution. The T4OL concentrations of the samples were determined by RP-HPLC.

\section{T4OL release kinetics}

To describe the drug release kinetics of the test formulations, zero-order (i.e., the amount of T4OL released per diffusion area vs. time), first-order (i.e., log of the amount of drug remaining to be released vs. time), Higuchi's (i.e., the amount of T4OL released per diffusional area vs. square root of time), and the Korsmeyer-Peppas theoretical models were used. The Korsmeyer-Peppas model ${ }^{24,25}$ is based on Equation 1:

$$
M_{\mathrm{t}} / M_{\infty}=K t^{n}
$$

where $M_{\mathrm{t}} / M_{\infty}$ is the fraction of drug released in a given time $t, n$ is the release exponent, and $K$ is the release factor. Plots of $\log \left(M_{\mathrm{t}} / M_{\infty}\right)$ as a function of $\log t$ provided the $n$ and $K\left(\mathrm{~h}^{-n}\right)$ values.

\section{RESULTS AND DISCUSSION}

The zein NPs obtained had average diameters that ranged from 120 to $190 \mathrm{~nm}$ and polydispersity index (PDI) values between 0.09 and 0.32. NPs with T4OL encapsulated can be observed in Figure $1 \mathrm{~A}$ and are in the form of dense spheres (with or without T4OL).

To optimize the experimental conditions used to prepare NPs with encapsulated T4OL, a $3^{3-1}$ fractional factorial design with quadruplicate samples at the central point was constructed with zein, T4OL, and TTA considered as factors and particle size, $P D I$, zeta potential, and $E E$ considered as responses (Table 1). The interpretation of the results was simplified by considering a response function $\left(R_{\mathrm{f}}\right)$ simultaneously associated with the four responses through Equation 2 , which was implemented to provide a higher response, i.e., $R_{\mathrm{f}}$ was

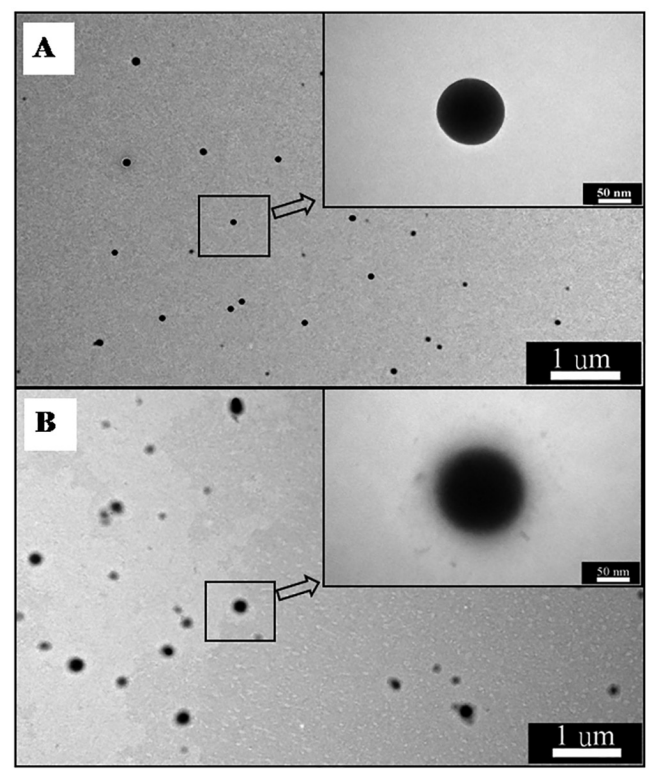

Figure 1. TEM images of zein NPs with encapsulated (A) T4OL and (B) T4OL + propylene glycol

fitted such that the highest value obtained represented the best global response of the system:

$$
R_{f}=\frac{(\text { size }+E E)}{\left(P D I^{*} z e t a\right)}
$$

In the present case, in terms of the response, experiment 7 provided the highest value, whereas experiment 2 gave the lowest value.

Table 1. Factors, levels, and responses for the $3^{3-1}$ fractional factorial design

\begin{tabular}{ccccccccccc}
\hline \multirow{2}{*}{ Issue } & \multicolumn{3}{c}{ Factors } & & \multicolumn{5}{c}{ Individual Responses } & \multirow{2}{*}{$\mathrm{R}_{\mathrm{f}}$} \\
\cline { 2 - 3 } \cline { 8 - 9 } & Zein & T4OL & TTA & & Size & PDI & Zeta & EE & \\
\hline 1 & 20 & 10 & 0.1 & & 183.9 & 0.15 & 33.5 & 65.6 & 49.65 \\
2 & 20 & 20 & 0.5 & & 175.2 & 0.32 & 57.7 & 66.6 & 13.09 \\
3 & 20 & 30 & 0.3 & & 119.1 & 0.18 & 48.5 & 86.1 & 23.50 \\
4 & 35 & 10 & 0.5 & & 134.3 & 0.22 & 55.1 & 82.5 & 17.88 \\
5 & 35 & 20 & 0.3 & & 171.9 & 0.11 & 46.1 & 78.1 & 49.30 \\
6 & 35 & 30 & 0.1 & & 168.8 & 0.15 & 45.7 & 84.9 & 37.01 \\
7 & 50 & 10 & 0.3 & & 184.6 & 0.09 & 43.3 & 93.5 & 71.36 \\
8 & 50 & 20 & 0.1 & & 179.0 & 0.12 & 48.5 & 85.7 & 45.48 \\
9 & 50 & 30 & 0.5 & & 191.1 & 0.09 & 45.0 & 93.2 & 70.20 \\
10 & 35 & 20 & 0.3 & & 172.9 & 0.10 & 46.5 & 78.6 & 54.08 \\
11 & 35 & 20 & 0.3 & & 174.9 & 0.15 & 44.1 & 80.0 & 38.53 \\
12 & 35 & 20 & 0.3 & & 168.9 & 0.16 & 45.1 & 77.9 & 34.20 \\
\hline
\end{tabular}

Table 2 shows the regression coefficient obtained for the $3^{3-1}$ fractional factorial design with quadruplicate samples at the central point. According to the statistical analysis, the TTA factor (linear and quadratic) and first-order zein*T4OL interaction were significant within the $95 \%$ confidence interval $(p>0.05)$. A lack of fit was not verified for the model within the $95 \%$ confidence interval because the lack of fit test provided a value of $p=0.22$ (ANOVA). Therefore, a response surface was obtained for zein and T4OL (Figure 2A); this response surface indicated that the response increased when both factors were maintained at a high level. However, the response (Figure 
2B) obtained for TTA and T4OL increased when TTA was fixed at the central point and T4OL was fixed at the low or high level. On the basis of our analysis of the response surface, zein at a concentration of 50 $\mathrm{mg} \mathrm{mL} \mathrm{m}^{-1}$ and TTA fixed at $0.3 \%$ in the aqueous phase were selected as the experimental conditions to be applied to study the influence of the T4OL concentration on the stability of NPs and $E E$. The data in Table 3 show that the best results were obtained with 10 and $100 \%$ of T4OL. The system with $100 \%$ of T4OL was selected because of the need to use a large amount of T4OL, with a compromise between

Table 2. Statistical results obtained from the $3^{3-1}$ fractional factorial design

\begin{tabular}{lccc}
\hline Factors & $\begin{array}{c}\text { Regression } \\
\text { Coefficient }\end{array}$ & Std. Err. & p-value \\
\hline Constant & 158.117 & 43.06 & $0.0357^{*}$ \\
Zein & -5.446 & 2.17 & 0.098 \\
T4OL & -9.074 & 3.01 & 0.057 \\
TTA & 383.937 & 114.63 & $0.044^{*}$ \\
Zein*Zein & 0.056 & 0.03 & 0.125 \\
T4OL*T4OL & 0.107 & 0.06 & 0.169 \\
TTA*TTA & -682.900 & 188.45 & $0.036^{*}$ \\
Zein*T4OL & 0.133 & 0.04 & $0.044^{*}$ \\
\hline
\end{tabular}

*Factors considered significant.

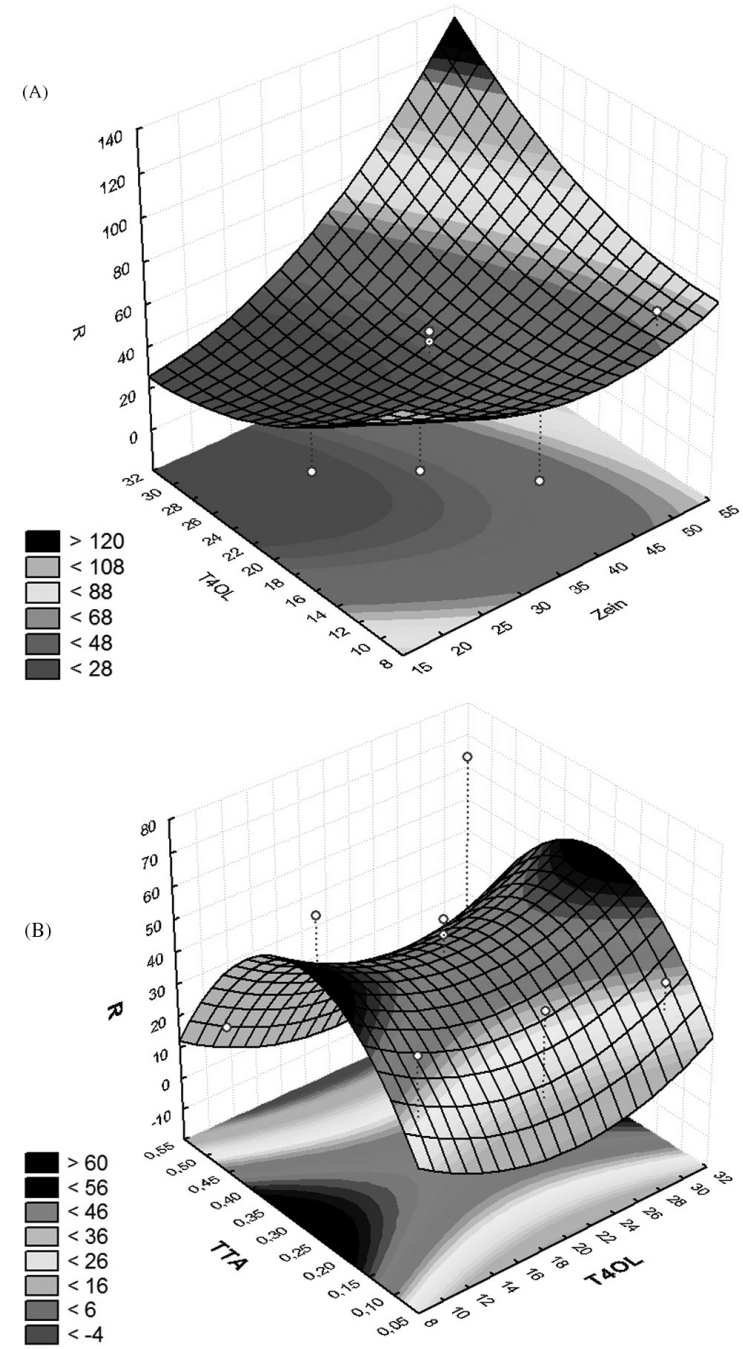

Figure 2. Response surfaces obtained for T4OL with (A) zein and (B) TTA the particle size, zeta potential, and $E E$. On the basis of the results, we conducted in vitro release assays using a system composed of zein $\left(50 \mathrm{mg} \mathrm{mL}^{-1}\right)$, T4OL $(100 \%)$, and TTA $(0.3 \%)$.

Table 3. Influence of the T4OL on the size, PDI, zeta potential, and EE of the formed zein NPs

\begin{tabular}{ccccc}
\hline$[\mathrm{T} 4 \mathrm{OL}] / \%$ & Size $/ \mathrm{nm}$ & PDI & $\begin{array}{c}\text { Zeta Potential } \\
/ \mathrm{mV}\end{array}$ & EE / \% \\
\hline 10 & $184.6 \pm 1.4^{\mathrm{a}}$ & $0.09 \pm 0.01^{\mathrm{a}}$ & $43.4 \pm 0.6^{\mathrm{c}}$ & $93.5 \pm 0.2^{\mathrm{c}}$ \\
20 & $196.0 \pm 2.6^{\mathrm{b}}$ & $0.11 \pm 0.01^{\mathrm{a}}$ & $36.2 \pm 1.5^{\mathrm{a}, \mathrm{b}}$ & $78.7 \pm 0.7^{\mathrm{b}}$ \\
40 & $185.5 \pm 3.2^{\mathrm{a}}$ & $0.12 \pm 0.01^{\mathrm{a}}$ & $37.5 \pm 1.6^{\mathrm{a}, \mathrm{b}}$ & $83.5 \pm 0.6^{\mathrm{a}}$ \\
60 & $182.7 \pm 2.1^{\mathrm{a}}$ & $0.11 \pm 0.01^{\mathrm{a}}$ & $40.3 \pm 0.6^{\mathrm{b}, \mathrm{c}}$ & $83.1 \pm 0.9^{\mathrm{a}, \mathrm{b}}$ \\
80 & $181.9 \pm 3.0^{\mathrm{a}}$ & $0.12 \pm 0.01^{\mathrm{a}}$ & $34.4 \pm 1.6^{\mathrm{a}}$ & $81.0 \pm 1.3^{\mathrm{a}}$ \\
100 & $185.1 \pm 2.4^{\mathrm{a}}$ & $0,10 \pm 0.01^{\mathrm{a}}$ & $39.7 \pm 2.0^{\mathrm{a}, \mathrm{b}, \mathrm{c}}$ & $91.4 \pm 0.6^{\mathrm{c}}$ \\
\hline
\end{tabular}

Same letters in the same column mean that no significant differences between samples $(\mathrm{p}<0,05)$.

Release studies are helpful tools in the early stages of the development of nanostructured systems because they can indicate whether the drug delivery is controlled/sustained or immediate and can also provide information on the type of interactions between the drug and excipients. In the development of drug delivery systems, the release behavior is a key factor in establishing their applicability. ${ }^{21}$

Formulations containing only zein and T4OL released $55.9 \%$ of the drug in $7 \mathrm{~h}$ (Figure 3). Oleic acid-based NPs exhibited higher release values compared with those that contained limonene $(36.5 \%$ vs. $27.9 \%$ in 7 h). Propylene glycol-containing NPs exhibited the lowest release value among the tested formulations (19.6\% in $7 \mathrm{~h}$ ). These results indicate the suitability of the formulations for sustained topical drug delivery.

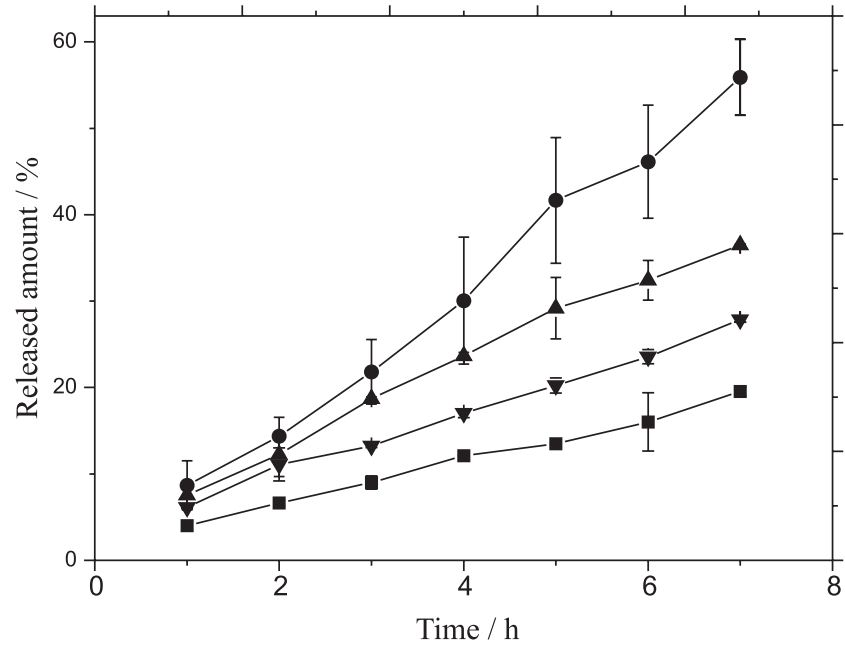

Figure 3. Release of T4OL from zein NPs in the absence $(\bullet)$ and presence of oleic acid $(\mathbf{\Lambda})$, limonene $(\mathbf{\nabla})$, and propylene glycol $(\mathbf{\square})$

The data obtained from in vitro release studies were fitted to various kinetic equations to determine the mechanism of T4OL release and the release rate. The criterion for selecting the most appropriate model was based on linear regression analysis $\left(>R^{2}\right)$. The constants obtained and corresponding $R^{2}$ values are shown in Table 4 . The zero-order kinetic equation is a model that explains well the behavior of T4OL release from the different formulations, as indicated by the obtained $R^{2}$ values being greater than those that resulted from the fits with the first-order and Higuchi's equations. According to the zero-order model, the drug release occurs at a constant rate and is 
Table 4. Results of model fitting for the test products

\begin{tabular}{|c|c|c|c|c|c|c|c|c|c|}
\hline \multirow{2}{*}{ Formulation } & \multicolumn{2}{|c|}{ Zero order } & \multicolumn{2}{|c|}{ First order } & \multicolumn{2}{|c|}{ Higuchi model } & \multicolumn{3}{|c|}{ Korsmeyer-Peppas } \\
\hline & $K\left(\mathrm{~h}^{-1}\right)$ & $R^{2}$ & $K\left(\mathrm{~h}^{-1}\right)$ & $R^{2}$ & $K\left(\mathrm{~h}^{-1 / 2}\right)$ & $R^{2}$ & $K\left(\mathrm{~h}^{-\mathrm{n}}\right)$ & $n$ & $R^{2}$ \\
\hline Zein+T4OL (1) & 8.04 & 0.9893 & 0.14 & 0.9621 & 0.45 & 0.9766 & 7.96 & 0.98 & 0.9875 \\
\hline 1+Propylene glycol & 2.50 & 0.9950 & 0.11 & 0.9433 & 0.22 & 0.9773 & 3.88 & 0.80 & 0.9939 \\
\hline 1+Oleic acid & 4.92 & 0.9915 & 0.11 & 0.9249 & 0.11 & 0.9917 & 7.34 & 0.83 & 0.9954 \\
\hline 1+Limonene & 3.47 & 0.9962 & 0.10 & 0.9423 & 0.16 & 0.9790 & 6.16 & 0.75 & 0.9913 \\
\hline
\end{tabular}

independent of the T4OL concentration. This constant rate is highly desirable with respect to drug release because a predictable amount of drug is released per unit of time under this condition. Table 4 also shows the results for the fitting of the data using the KorsmeyerPeppas model. This model is typically applied in the analysis of release data related to polymeric systems in cases where the release mechanism is not well understood or when more than one type of drug release phenomenon occurs. ${ }^{26}$ Equation 1 provided a good fit with the experimental data, and the $n$ values obtained reflected the two different situations, i.e., the absence and the presence of the SPEs. In the case of the Korsmeyer-Peppas model the $n$ value is used to characterize different release mechanisms. ${ }^{26-28}$ A value of $n=0.5$ indicates drug release through Fickian diffusion, whereas $0.5<n<1$ indicates that the mass transfer follows a non-Fickian model, which is known as anomalous transport. Zero-order drug release or Case-II transport is associated with an $n$ value of 1 . For $n>1$, the mechanism of drug release is regarded as super Case-II transport.

The $n$ value obtained for the T4OL encapsulated in the zein NPs was approximately 1.0 in the absence of the SPEs. Notably, the $K$ values obtained using the zero-order and Korsmeyer-Peppas models were very similar (8.04 and $7.96 \mathrm{~h}^{-1}$, respectively). Thus, the Korsmeyer-Peppas model suggests that the release of the T4OL under these conditions follows zero-order kinetics. With the addition of SPEs to NPs, the $n$ values changed considerably (0.75-0.83). These results suggest that the addition of SPEs changes the mass transfer from zero-order to the anomalous transport of the non-Fickian model, which entails mixed behavior involving diffusion and relaxation of the polymeric chains.

The solubility of T4OL in the systems containing SPEs follows the order: propylene glycol $>R-(+)$-limonene $>$ oleic acid, whereas the release order of T4OL in NPs was: without SPE $>$ oleic acid $>$ $R$-(+)-limonene $>$ propylene glycol (Figure 3 ). The release order observed for T4OL $(\log \mathrm{P}=2.99)^{29}$ in the presence of the SPEs reflects the lipophilicity: oleic acid $(\log \mathrm{P}=6.5)^{30}>R$-(+)-limonene $(\log \mathrm{P}=$ $4.23)^{30}>$ propylene glycol $(\log \mathrm{P}=-0.78) .{ }^{31}$ When a compound with a high lipophilicity, such as oleic acid, is added to the system it can preferentially interact with the zein rather than the T4OL, resulting in the faster release of T4OL. However, $R-(+)$-limonene exhibits lower lipophilicity, and its interaction with zein tends to be weaker, which increases the T4OL-zein interaction. When propylene glycol (a hydrophilic compound) is used, coated NPs are formed because of the interaction of this compound with the NP surface through hydrogen bonding, and this hydrophilic coating on the NP surface has a retardant effect of the T4OL release (Figure 1B).

The results showed that although the zein concentration has a pronounced effect on the $E E$ in a direct ratio, its apparent solubility is limited to $50 \mathrm{mg} \mathrm{mL}^{-1}$ in $87 \%$ ethanol. To evaluate the influence of the solvent composition on the particle size and PDI of zein NPs, various proportions of ethanol/water were used to dissolve the zein. ${ }^{32}$ At high zein concentrations, the formation of aggregates occurred, which is undesirable in relation to their application in biopharmaceutics because the formation of aggregates can reduce the activity and solubility as well as induce immunogenicity and other adverse reactions. ${ }^{33}$ This phenomenon is common in protein solutions because protein solubility can be strongly affected by the $\mathrm{pH}$ and ionic strength of the medium. ${ }^{34}$

The zein NPs prepared in this study exhibited a slow T4OL release rate, which is of interest because the skin behaves as a natural barrier that protects the body against the external environment and the permeation of substances. ${ }^{3}$ Therefore, in the case of slow release, the active compound will have sufficient time to penetrate into the skin and into the cells. Moreover, NP formulations used in dermatological products can follow intercellular, transcellular, or transappendageal pathways during skin permeation. ${ }^{35}$ However, for the treatment of cancer cells, NPs should remain in the cells, and previous studies have shown that zein NPs can easily be internalized into living cells. ${ }^{15}$ The optimized system has potential uses in anti-melanoma therapy because it offers sustained delivery and because zein-based systems exhibit rapid cell internalization. ${ }^{15}$

\section{CONCLUSIONS}

Zein NPs were successfully prepared using an adapted antisolvent precipitation method and were obtained in the form of dense spheres. Several factors, such as the concentrations of surfactant, T4OL, and zein, were changed during the preparation of NPs to optimize the conditions to obtain a monodisperse and stable nanostructured system. The system studied exhibited a high $E E$ value (91\%). In vitro release experiments showed the sustained release of T4OL with zero-order kinetics. This result was corroborated by analysis conducted using the Korsmeyer-Peppas model. The addition of SPEs caused a change in the T4OL release toward a nonFickian transport model, with mixed behavior involving diffusion and relaxation of the polymeric chains. The results indicate that the zein NPs studied here are promising nanostructured systems for the prolonged delivery of T4OL and are potential useful in anti-melanoma therapy. The skin permeation and drug activity are currently the subject of further investigations designed to verify the application of this system to a real situation. Stability studies are also in progress.

\section{ACKNOWLEDGMENTS}

The financial support of the Brazilian government agency Conselho Nacional de Desenvolvimento Científico e Tecnológico (CNPq) and UFSC is gratefully acknowledged.

\section{REFERENCES}

1. Schadendorf, D.; Worm, M.; Algermissen, B.; Kohlmus, C. M.; Czarnetzki, B. M.; Cancer 1994, 73, 103.

2. Kline, A. J.; University of Missouri-Columbia, United States, 2005.

3. Reichling, J.; Landvatter, U.; Wagner, H.; Kostka, K.-H.; Schaefer, U. F.; Eur. J. Pharm. Biopharm. 2006, 64, 222. 
4. Calcabrini, A.; Stringaro, A.; Toccacieli, L.; Meschini, S.; Marra, M.; Colone, M.; Salvatore, G.; Mondello, F.; Arancia, G.; Molinari, A.; J. Invest. Dermatol. 2004, 122, 349.

5. Giordani, C.; Molinari, A.; Toccacieli, L.; Calcabrini, A.; Stringaro, A.; Chistolini, P.; Arancia, G.; Diociaiuti, M.; J. Med. Chem. 2006, 49, 4581.

6. Alexander, A.; Dwivedi, S.; Giri, T. K.; Saraf, S.; Saraf, S.; Tripathi, D. K.; J. Controlled Release 2012, 164, 26.

7. Larkins, B. A.; Hurkman, W. J.; Plant Physiol. 1978, 62, 256.

8. Wang, Y.; Padua, G. W.; Langmuir 2010, 26, 12897.

9. Reddy, N.; Yang, Y.; Trends in Biotechnol. 2011, 29, 490.

10. Lai, L. F.; Guo, H. X.; Int. J. of Pharm. 2011, 404, 317.

11. Wu, Y.; Luo, Y.; Wang, Q.; LWT Food Sci. and Technol. 2012, 48, 283.

12. Hu, D.; Lin, C.; Liu, L.; Li, S.; Zhao, Y.; J. Food Eng. 2012, 109, 545.

13. Patel, A. R.; Heussen, P. C. M.; Hazekamp, J.; Drost, E.; Velikov, K. P. Food Chem. 2012, 133, 423.

14. Zou, T.; Li, Z.; Percival, S. S.; Bonard, S.; Gu, L. Food Hydrocolloids 2012, 27, 293.

15. Regier, M. C.; Taylor, J. D.; Borcyk, T.; Yang, Y.; Pannier, A.; J. Nanobiotechnol. 2012, 10, 1.

16. Soppimath, K. S.; Aminabhavi, T. M.; Kulkarni, A. R.; Rudzinski, W. E.; J. Controlled Release 2001, 70, 1.

17. Fayad, S. J.; Zanetti-Ramos, B. G.; Barreto, P. L.; Soldi, V.; Minatti, E.; J. Braz. Chem. Soc. 2013, 24, 1012.

18. Bazzo, G. C.; Lemos-Senna, E.; Gonçalves, M.; Pires, A. T. N.; J. Braz. Chem. Soc. 2008, 19, 914.

19. Choi, W. I.; Lee, J. H.; Kim, J.-Y.; Kim, J.-C.; Kim, Y. H.; Tae, G.; J. Controlled Release 2012, 157, 272.
20. Luo, Y.; Zhang, B.; Whent, M.; Yu, L. L.; Wang, Q.; Colloids Surf., B 2011, 85, 145.

21. Sousa, F.; Luzardo-Álvarez, A.; Blanco-Méndez, J.; Martín-Pastor, M.; Int. J. Pharm. 2012, 439, 41.

22. Barry, B.; Adv. Drug Delivery Rev. 2002, 54, S31.

23. Xu, H.; Jiang, Q.; Reddy, N.;Yang, Y.; J. Mater. Chem. 2011, 21, 18227.

24. Mello, V. A.; Ricci-Júnior, E.; Quim. Nova 2011, 34, 933.

25. Korsmeyer, R. W.; Gurny, R.; Doelker, E. M.; Buri, P.; Peppas, N. A.; Int. J. Pharm. 1983, 15, 25.

26. Costa, P.; Lobo, J. M. S. Eur. J. Pharm. Sci. 2001, 13,123.

27. Mathew, S. T.; Devi, S. G.; Sandhya, K. V.; AAPS PharmSciTech 2007, 8, E1.

28. Peppas, N. A.; Pharm. Acta Helv. 1985, 60, 110

29. Zasshi, Y.; J. Pharm. Soc. Jpn. 2006, 126, 307.

30. del Rio-Sancho, S.; Serna-Jimenez, C.; Calatayud-Pascual, M.; Balaguer-Fernandez, C.; Femenia-Font, A.; Merino, V.; López-Castellano, A.; Eur. J. Pharm. Biopharm. 2012, 82, 164.

31. Duracher, L.; Blasco, L.; Hubaud, J.-C.; Vian, L.; Marti-Mestres, G.; Int. J. Pharm. 2009, 374, 39.

32. Kim, S.; Xu, J.; J. Cereal Sci. 2008, 47, 1.

33. Pan, H.; Chen, K.; Pulisic, M.; Apostol, I.; Huang, G.; Anal. Biochem. 2009, 388, 273 .

34. Ryan, K.; Vardhanabhuti, B.; Jaramillo, D.; van Zanten, J.; Coupland, J.; Foegeding, E.; Food Hydrocolloids 2012, 27, 411.

35. Desai, P. R.; Shah, P. P.; Hayden, P.; Singh, M.; Pharm. Res. 2013, 30, 1037. 